Engineering \& Technology | Luis Fernando Velásquez-García

\title{
Bringing space closer with 3D printing
}

To fulfil the promise of the "NewSpace" revolution and open the door to commercia space exploration that is accessible to everyone, low cost space technologies are necessary. In order to lower the cost of spacecra Máximo from the Tecnológico deis Fernando VelásquezGarcia from the Massachusetts Institute of Technology report on the first additively manufactured (3D-printed) electrospray thrusters to be used in nanosatellites. These thrusters are not only cheaper and quicker to manfiracter, but they also use propello important development is a significant contribution to the democratization of nanosatellite propulsion technology.

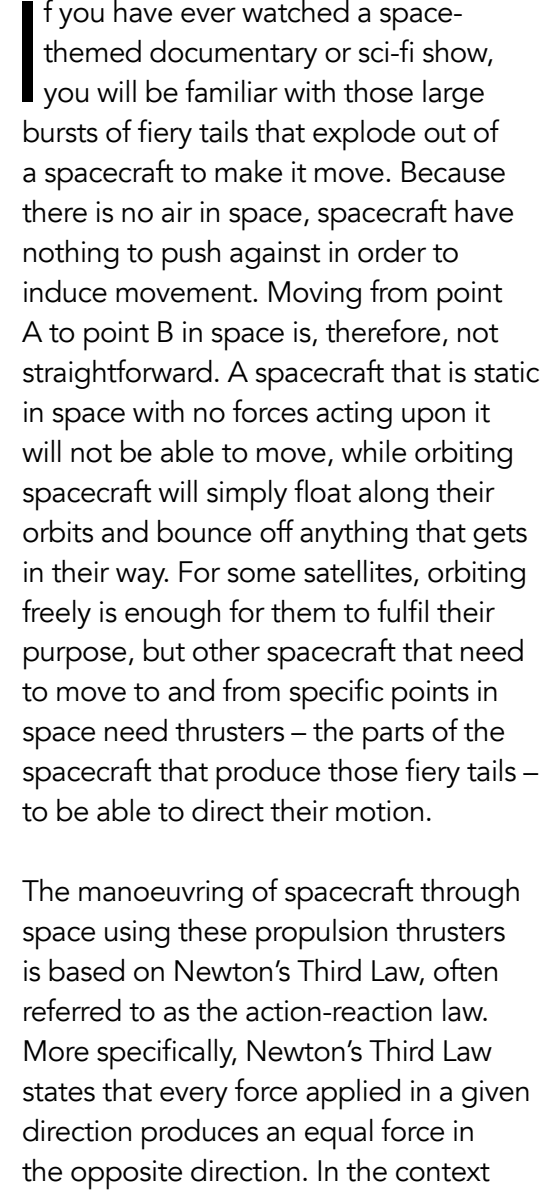

the opposite direction In the context

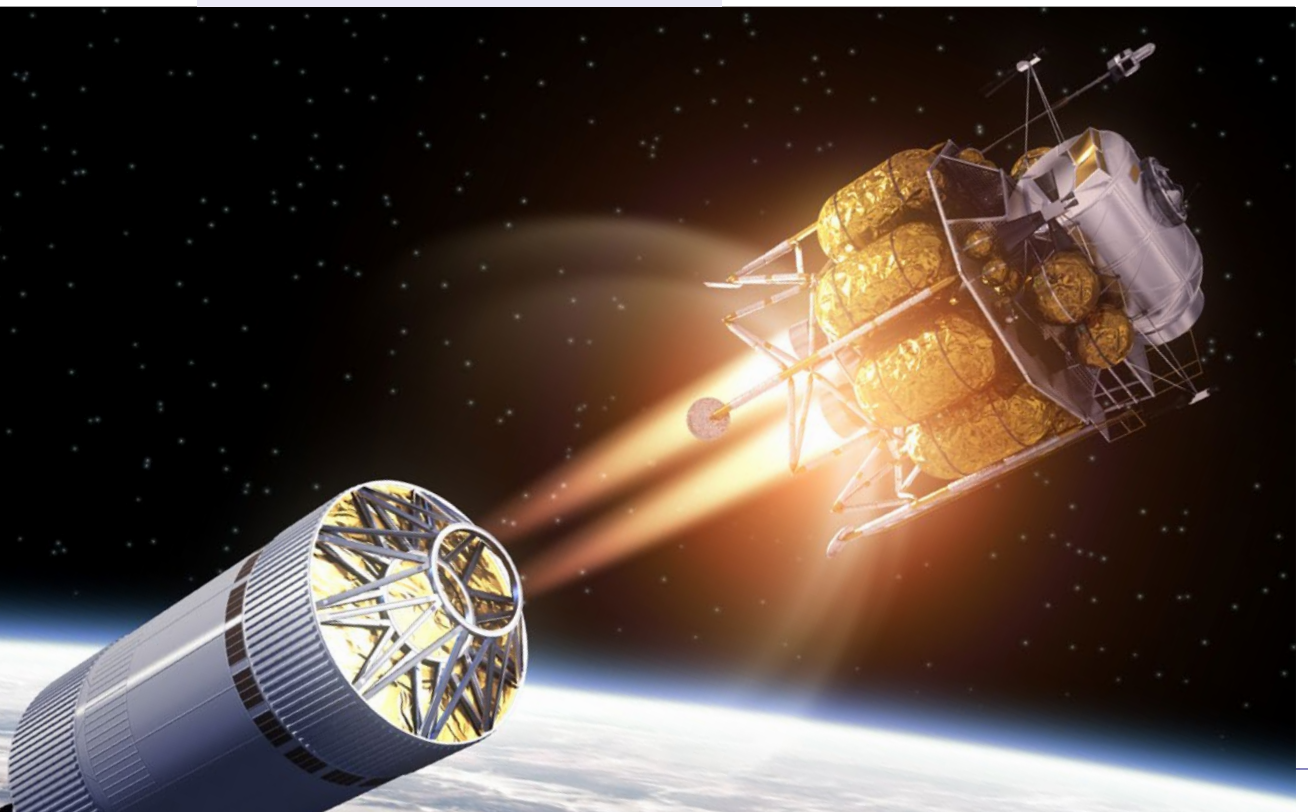

of spacecraft propulsion, the force generated by the violent expulsion of glowing plume by a thruster results in an equal force that pushes the spacecraft in the opposite direction. A single burst of the back thruster will propel the spacecraft forward, and the motion will

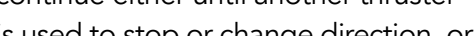
unt an obstap comes in the way of the spacecreft These basic rules apply for any device that requires directed motion in space, be it a larger spaceship with a crew or a small satellite sent into space to fulfil a specific purpose.

\section{PROPELLING NANOSATELLITES} INTO THE "NEWSPACE"

Space was once a drea countries raced to reach first, and that only a few countries had access to for a long time. However, since the first human travelled into the great unknown many other countries, agencies and (t) space. The concept of "NewSpace" has merged to referto a new wave of space to the growth of a commercial space industry. The interest in exploring space goes further than a desire to visit it: space offers many opportunities for applications on Earth, namely related to the Intern observation and monitoring, for example.

To put space and its potential applications within everyone's reach, and to allow for the fulfilment of the "NewSpace" revolution, the cost of space travel and related technology must be dramatically reduced. In particular, there are a number of target-focused expensive, (a) space. To this end, it is currently possible to manufacture nanosatellites weighing only $1-10 \mathrm{~kg}$. These small spacecraft a usually called "Cunesats", made up of shaped structure measuring $10 \mathrm{~cm}$ on each edge that weighs approximately $1 \mathrm{~kg}$. These nanosatellites need scaleddown propulsion engines that allow their controlled movement in space relatively low cost.

An attractive technology to propel and electrohydrodynamic jetting of charged particles from a liquid propellant. These rockets are called electrospray thrusters and can be engineered in a way th can be relatively easily scaled dow Importantly, the particles emitted by these electrohydrodynamic thrusters can be either positively or charged, allowing the charge neutrally by alternating the kind alternating the kind
of particles being emitted by the thruster. Electrospray thrusters can prope nanosatellites efficiently, consun less propellant

than competing technologies and are therefore more cost effective. Nevertheless, the manufacture of small-scale electrospray thrusters is very expensive and time-consumin hindering their use and the further Ill scale, low-cost space technology.

The manufacture of small-scale electrospray thrusters is very expensive and time-consuming, which hinders their use and the further development of

program, Melo-Máximo and Velásquez3D PRINTING THRUSTS SPACE TECHNOLOGY The NewSat program, a collaboration between Dr Luis Fernando VelásquezGarcia, Professor Wojciech Matus demonstrion of low-cost, additively 列 Be mployed as nanosatellite thrusters. With 25.4 millisters are square devices omm high, made of either polymer or metal, weighing under 21 grams, that sharp conic needles. A dense forest of thin nanowires, of 5-345 $\mathrm{nm}$ diameter is grown onto the squares creating and a consortium of Portuguese Selas and Nelson Ferreira from the company Stratosphere S.A., is exploring additive manufacturing methods $-3 D$ printing in other words - to demonstrate lownanosatellites. Working under this a nanostructured porous material that transports liquid between the propellant reservoir and the needle tips where, if a suitable bias voltage is applied (using as counter-electrode a metal plate with an array of apertures that faces the array of needles), emission of charged particles
takes place, producing thrust.
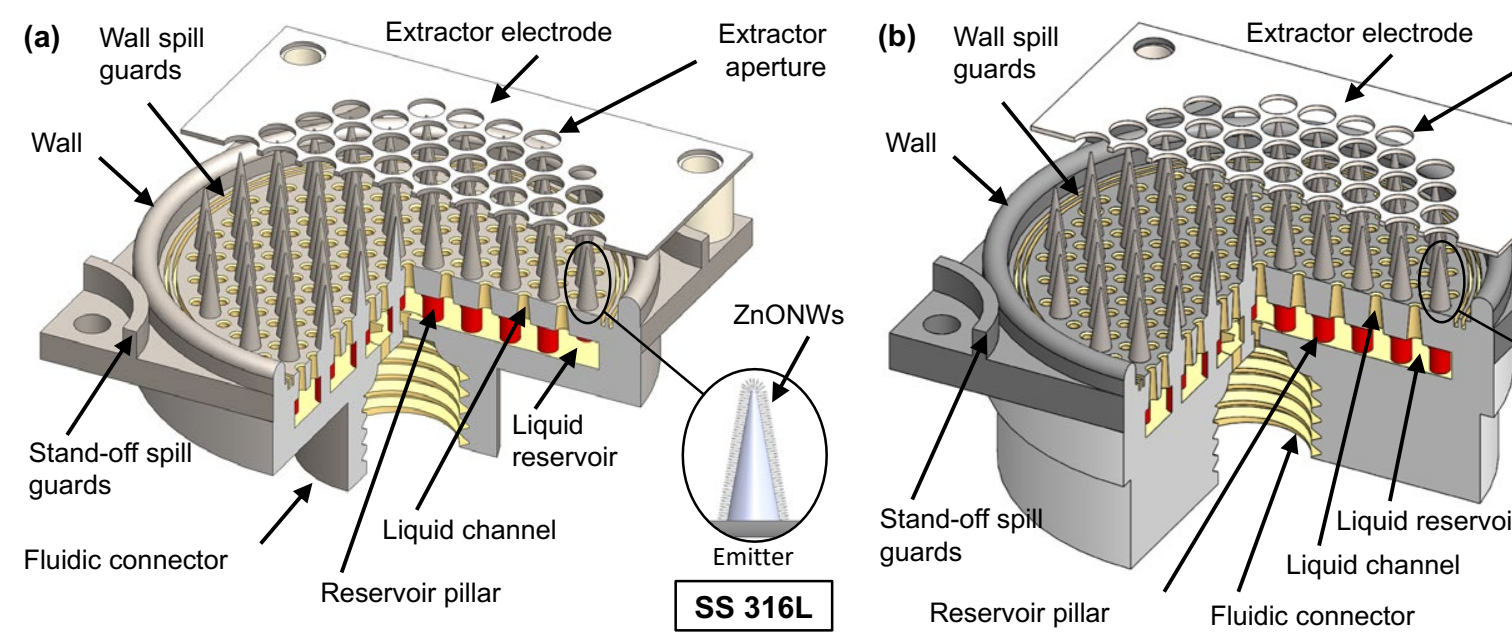

Extractor
aperture

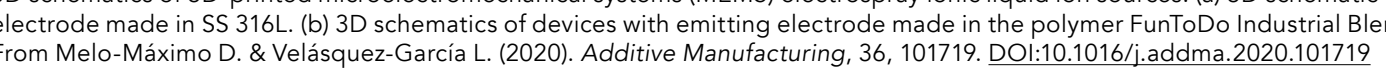




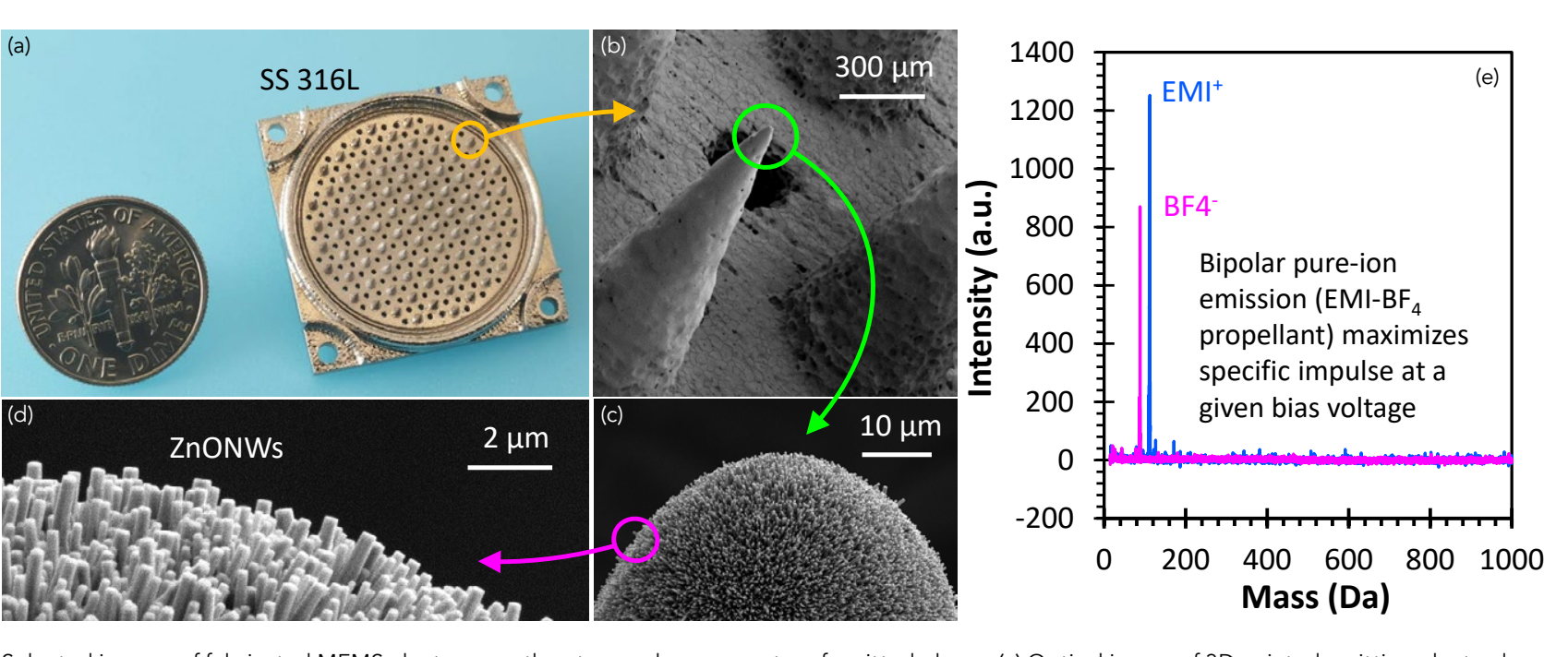

Selected images of fabricated MEMS electrospray thrusters and mass spectra of emitted plume. (a) Optical image of 3D-printed emitting electrode
made of SS $316 L$, (b) close-up SEM image of an emitter, (c) close-up SEM image of an emitter tip conformally coated with a dense zinc oxide nanowire

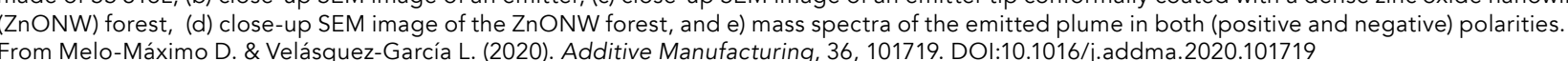

The choice of propellant is an important actor that affects thruster performan tandard (nvironnatal conditions) popular choice of electrospray thruster propellant. In this work, Melo-Máximo and Velásquez-García chose the ionic liquid 1-ethyl-3-methylimidazolium

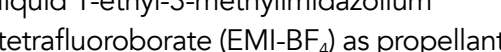
due to its high electrical conductivity and negligible vapor pressure. Importantly, EMII-BF ${ }_{4}$ also produces near-symmetric positive and negative plumes that facilitate attaining a spacecraft's charged neutrality

Another factor that affects electrospray thruster performance is the spread in the properties of the charged pestiven poully emit various types of charged particles, which significantly reduces the specific impulse of the thrustermeasure of how efficiently an engine uses propellant. On the other hand, the 3D-printed devices reported by Melo-Máximo and Velásquez-García are the very first electrospray thrusters or which pure-ion emission from ionic liquids was observed. This unique characteristic has resulted in a specific impulse for a given bias voltage that is in fact, higher than the current state-of the-art technology. This development is an outstanding advancement in space propulsion technology, since it savings, signicant propellonthid (a) used by the research team to produce thrusters reported in their study have advantages over the commonly employed precision subtractive manufacturing methods, such as laser machining and cleanroom microfabrication, which are very expensive and time-consuming The manufacturing methods used by

units to be cheaply and quickly produced. Furthermore, these thrusters and other devices developed by the VelásquezGarcía grou at MIT are examples of how multi-material additive manufacturing can implement complex hardware that is not feasible or impractical to create with other manufacturing methods and/or that can attain better performance than the state of the art. The remarkable results

This development is an outstanding advancement in space propulsion technology, since it allows for significant propellant fluid savings, and hence lower costs, without compromising performance. Melo-Máximo and Velásquez-García allow for the production of devices in a matter dollars per device (about two-orders-of magnitude reduction in both cases), white still being versatile and attaining high performance. Both the faster production and lower cost helps to shorten design iterations, since 3D printing allows for small- or medium-sized batches of test

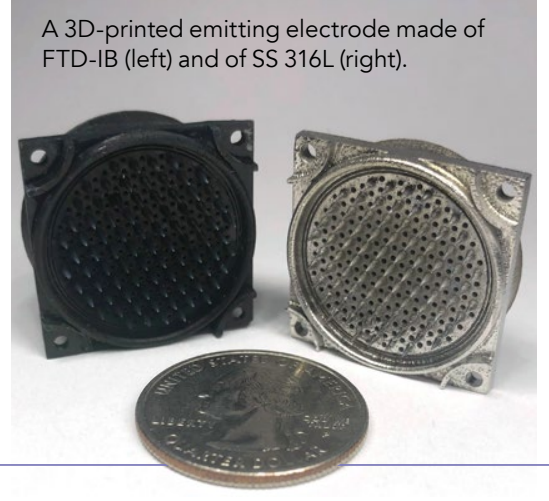

achieved by Melo-Máximo and VelásquezGarcía with their reported thrusters are to democratize nanosatellite propulsion technology and to expand access to the "NewSpace".

The work developed by Melo-Máximo and Velásquez-García was sponsored by the Monterrey Tec-Massachusetts Institute of Technology (MIT) Nanotechnology program and the NewSat project. The NewSat project, mentioned earlier, is co-funded by the Operational Program for Competitiveness and Internationalisation (COMPETE2020), Portugal 2020, the (EDC , and he Ponuguese Foundation the MIT Portugal program.
In addition, the $3 \mathrm{D}$ printing methods European Regional Development Fund

\section{Research Objectives}

Luis Fernando Velásquez-García's group uses micro and nanotechnology to conduct fundamental and applied research on systems that harness high electric field phenomena.

\section{Detail}

Address

achusetts Avenue

Office 39-415B

Cambridge MA 02139 USA

Bio

DrLuis Fernando Velásquez-García is a principal investigator with the Microsystems Technology Laboratories of the Massachusetts Institute of Technology. He leads a group that uses micro and nanotechnology to conduct fundamental and applied research on systems that harness high electric field phenomena, focusing on space, healthcare, energy, and manufacturing applications.

Funding

This work was sponsored by the Monterrey TecMassachusetts Institute of Technology (MIT) The Nechnology program and hew Program for Competitivenss and the Operational (COMPETE2020) Portugal 2020, the European Regional Development Fund (ERDF), and the Portuguese Foundation for Science and Technology (FTC) under the MIT Portugal program.

\section{Collaborators}

This research is part of the NewSat program that is exploring additive manufacturing to implement better and more capable nanosatellites. Newsat is a collaboration between Dr Luis Fernando Velásquez-García, Professor Wojciech Matusik (EECS), and Professor Maria Yang (MechE) from MIT, and a consortium of Portuguese institutions lead by Gustav

Massachusetts Institute of Technology

\section{References}

3D-printed nanosatellite electrospray thrusters: Melo-Máximo, D. and Velásquez-Garćá, LF. (2020). Additively manufactured electrohydrodynamic ionic liquid pure-ion sources for nanosatellite propulsion. Additive Manufacturing 36, 101719. DOl:10.1016/j.addma.2020.101719

Melo-Máximo DV, et al. (2000). Fully additively manufactured, nanostructure, miniature ionic liquid electrospray sources. Micro Electro Mechanical Systems (MEMS). DOI:10.1109/ MEMS46641.2020.9056405

Other complex, multi-material 3D-printed, miniaturized power conversion devices developed by the VelásquezGarcia group at MIT:

Taylor AP, et al. (2020). Compact, magnetically actuated, additively manufactured pumps for liquids and gases J. Phys.
D: Appl. Phys. 53 (2020) 355002 . DOl:10.1088 11361-6463/ ab8de8

Taylor AP, et al. (2019). Fully 3D-Printed, Monolithic, Mini Magnetic Actuators for Low-Cost, Compact Systems. J Microelectromechanical Sys. 28(3), 481-93. DOI:10.1109/ JMEMS.2019.2910215

Taylor AP and Velásquez-García LF (2017). Miniaturized diaphragm vacuum pump by multi-material additive

\section{Personal Response}

What type of spacecraft could these 3D printed ich missions are they to be on?

II The thrusters are intended to propel satellites. (the engine is an array of emitters, and the size of the array is arbitrary); therefore, arguably, large-area thruster panels could be developed to produce thrust levels of interest to a wide range of satellite sizes, from standard (large, school bus-sized) satelles to cubesats. The high specific impulse (eg. travelling to deep space, continuously correcting the spacecraft's orbit) because those are missions where the propellant savings that result from using a thruster with
high specific impulse would make the most difference. 